\title{
Management of Classic Ulcerative Pyoderma Gangrenosum
}

\author{
Ladan Afifi, MD, MS; Alex G. Ortega-Loayza, MD, MCR; Kanade Shinkai, MD, PhD
}

\section{PRACTICE POINTS}

- The diagnosis of pyoderma gangrenosum (PG) poses a challenge in clinical practice that could be minimized by following a stepwise algorithm based on initial test results (including skin biopsies) and features of the patient's clinical presentation.

- As there is no US Food and Drug Administrationapproved treatment for PG, a stepwise algorithm approach in combination with the clinical experience addressing inflammation and wound care is essential to reach control and remission of PG.

Pyoderma gangrenosum (PG) is a rare neutrophilic dermatosis with unclear etiology and is associated with notable morbidity. Due to the rarity of PG, there are limited large, multicentered, randomized trials to guide management. We aim to highlight best practices in $P G$ management through survey responses from expert medical dermatologists.

Cutis. 2020;106:119-123.

$\square$ yoderma gangrenosum (PG) is a rare, chronic, ulcerative, neutrophilic dermatosis of unclear etiology. Large, multicentered, randomized controlled trials (RCTs) are challenging due to the rarity of PG and the lack of a diagnostic confirmatory test; therefore, evidencebased guidelines for diagnosis and treatment are not well established. Current management of PG primarily is guided by case series, small clinical trials, and expert opinion..$^{1-4}$ We conducted a survey of expert medical dermatologists to highlight best practices in diagnostic and therapeutic approaches to PG.

\section{Methods}

The Society of Dermatology Hospitalists (SDH) Scientific Task Force gathered expert opinions from members of the SDH and Rheumatologic Dermatology Society (RDS) regarding PG workup and treatment through an online survey of 15 items (eTable 1). Subscribers of the SDH and RDS LISTSERVs were invited via email to participate in the survey from January 2016 to February 2016. Anonymous survey responses were collected and collated using SurveyMonkey. The survey results identified expert recommendations for evaluation, diagnosis, and treatment of PG and are reported as the sum of the percentage of respondents who answered always (almost 100\% of the time) or often (more than half the time) following a particular course of action. A subanalysis was performed defining 2 groups of respondents based on the number of cases of PG treated per year $(\geq 10$ vs $<10)$. Survey responses between each group were compared using $\chi^{2}$ analysis with statistical significance set at $P=.05$.

\section{Results}

Fifty-one respondents completed the survey out of 140 surveyed (36\% response rate). All respondents were

Dr. Afifi is from the Department of Dermatology, University of California, Los Angeles. Dr. Ortega-Loayza is from the Department of Dermatology, Oregon Health \& Science University, Portland. Dr. Shinkai is from the Department of Dermatology, University of California, San Francisco.

The authors report no conflict of interest.

This consensus activity was granted institutional review board exemption status by the University of California, San Francisco Committee on Human Research.

The opinions expressed in this article were presented in part at the American Academy of Dermatology Annual Meeting; March 4-7, 2016; Washington, DC.

The eTables are available in the Appendix online at www.mdedge.com/dermatology.

Correspondence: Kanade Shinkai, MD, PhD, 1701 Divisadero St, 3rd Floor, San Francisco, CA 94115 (Kanade.shinkai@ucsf.edu). doi:10.12788/cutis.0076 
dermatologists, and 96\% (49/51) were affiliated with an academic institution. Among the respondents, the number of PG cases managed per year ranged from 2 to 35 .

Respondents consistently ordered skin biopsies (92\% [47/51]) and tissue cultures (90\% [46/51]), as well as certain ancillary tests, including complete blood cell count $(96 \%$ [49/51]), complete metabolic panel $(86 \%$ [44/51]), serum protein electrophoresis (76\% [39/51]), and hepatitis panel $(71 \%$ [36/51]). Other frequently ordered studies were rheumatoid factor $(69 \%$ [35/51]), antinuclear antibodies (67\% [34/51]), and antineutrophilic antibodies (65\% [33/51]). Respondents frequently ordered erythrocyte sedimentation rate $(59 \%$ [30/51]), C-reactive protein (55\% [28/51]), cryoglobulins $(53 \%$ [27/51]), urine protein electrophoresis (53\% [27/51]), hypercoagulability workup (49\% [25/51]), and serum immunofixation test $(49 \%$ [25/51]). Human immunodeficiency virus testing (43\% [22/51]), chest radiograph (41\% [21/51]), colonoscopy (41\% [21/51]) and referral to other specialties for workup-gastroenterology (38\% [19/51]), hematology/oncology (14\% [7/51]), and rheumatology $(10 \%$ [5/51]) - were less frequently ordered (eTable 2).

Systemic corticosteroids were reported as first-line therapy by most respondents $(94 \%$ [48/51]), followed by topical immunomodulatory therapies $(63 \%$ [32/51]). Topical corticosteroids (75\% [38/51]) were the most common first-line topical agents. Thirty-nine percent of respondents (20/51) prescribed topical calcineurin inhibitors as first-line topical therapy. Additional therapies frequently used included systemic cyclosporine (47\% [24/51]), antineutrophilic agents (41\% [21/51]), and biologic agents $(37 \%$ [19/51]). Fifty-seven percent of respondents (29/51) supported using combination topical and systemic therapy (Table).

A wide variety of wound care practices were reported in the management of PG. Seventy-six percent of respondents (39/51) favored petroleum-impregnated gauze, 69\% (35/51) used nonadhesive dressings, and $43 \%(22 / 51)$ added antimicrobial therapy for PG wound care (eTable 3 ). In the subanalysis, there were no significant differences in the majority of answer responses in patients treating 10 or more PG cases per year vs fewer than 10 PG cases, except with regard to the practice of combination therapy. Those treating more than 10 cases of PG per year more frequently reported use of combination therapies compared to respondents treating fewer than 10 cases $(P=.04)$.

\section{Comment}

Skin biopsies and tissue cultures were strongly recommended (>90\% survey respondents) for the initial evaluation of lesions suspected to be PG to evaluate for typical histopathologic changes that appear early in the disease, to rule out PG mimickers such as infectious or vascular causes, and to prevent the detrimental effects of inappropriate treatment and delayed diagnosis. ${ }^{5}$

\section{Pyoderma Gangrenosum \\ Treatment $(\mathrm{N}=51)$}

Reported Use, ${ }^{a}$

n (\%)

Treatment

\begin{tabular}{lc} 
Systemic therapy & $48(94)$ \\
\hline Topical immunomodulatory therapy & $32(63)$ \\
\hline
\end{tabular}

Intralesional therapy

First-line combination therapy approach

Combination of topical and systemic treatments

$24(47)$

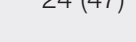

Combination of topical, intralesional, 19 (37)

and systemic treatments

Combination of intralesional and $11(22)$

systemic treatments

Do not use combination approach 10 (20)

Combination of topical and 8 (16)

intralesional treatments

First-line topical therapy

Topical corticosteroids $38(75)$

\begin{tabular}{ll}
\hline Topical calcineurin inhibitors & $20(39)$ \\
\hline Dapsone gel & $5(10)$ \\
\hline Do not use topical therapy & $5(10)$ \\
\hline Other & $2(4)$
\end{tabular}

First-line systemic therapy

Corticosteroids 47 (92)

Cyclosporine $24(47)$

Antineutrophilic agents (eg, 21 (41)

dapsone, colchicine, sulfasalazine)

Biologics (eg, infliximab, etanercept, 19 (37) adalimumab, ustekinumab)

\begin{tabular}{ll}
\hline Mycophenolate mofetil & $14(27)$ \\
\hline Azathioprine & $4(8)$ \\
\hline Intravenous immunoglobulin & $2(4)$ \\
\hline $\begin{array}{l}\text { IL-1 inhibitors (eg, anakinra, } \\
\text { canakinumab) }\end{array}$ & $0(0)$ \\
\hline $\begin{array}{l}\text { Cyclophosphamide } \\
\text { (0) }\end{array}$ & $0(0)$
\end{tabular}

${ }^{a}$ Data represent an aggregate of respondents who answered always use (almost 100\% of the time) and often use (more than half the time). 
Suspected PG warrants a reasonable search for related conditions because more than $50 \%$ of PG cases are associated with comorbidities such as rheumatoid arthritis, inflammatory bowel disease, and hematologic disease/ malignancy. ${ }^{6,7}$ A complete blood cell count and comprehensive metabolic panel were recommended by most respondents, aiding in the preliminary screening for hematologic and infectious causes as well as detecting liver and kidney dysfunction associated with systemic conditions. Additionally, exclusion of infection or malignancy may be particularly important if the patient will undergo systemic immunosuppression. In challenging PG cases when initial findings are inconclusive and the clinical presentation does not direct workup (eg, colonoscopy to evaluate gastrointestinal tract symptoms), serum protein electrophoresis, hepatitis panel, rheumatoid factor, antinuclear antibodies, and antineutrophilic antibody tests also were frequently ordered by respondents to further evaluate for underlying or associated conditions.

This consensus regarding skin biopsies and certain ancillary tests is consistent with the proposed diagnostic criteria for classic ulcerative PG in which the absence or exclusion of other relevant causes of cutaneous ulcers is required based on the criteria. ${ }^{8}$ The importance of ensuring an accurate diagnosis is paramount, as a $10 \%$ misdiagnosis rate has been documented in the literature. ${ }^{5}$

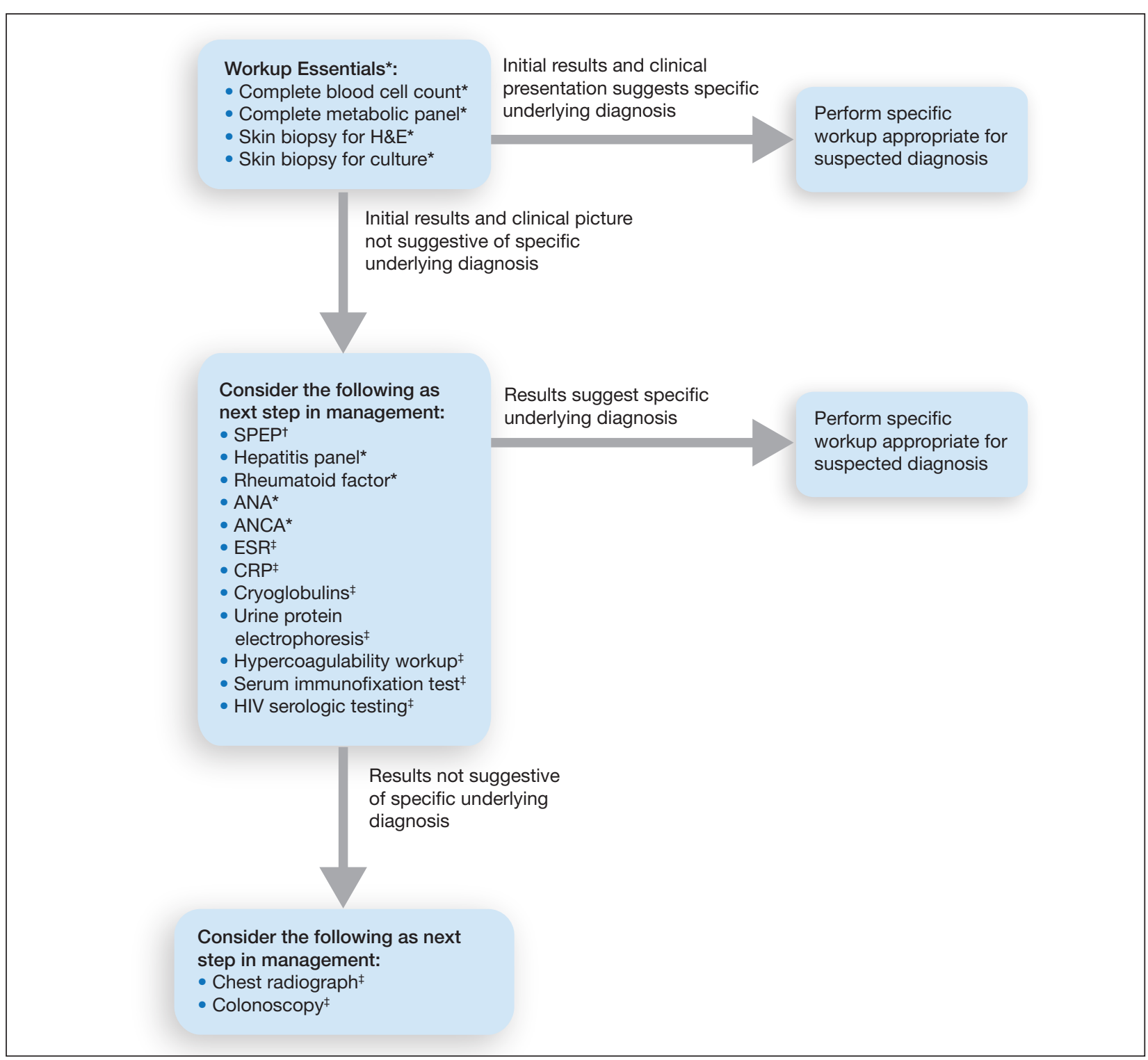

FIGURE 1. Proposed stepwise algorithm of classic ulcerative pyoderma gangrenosum workup. H\&E indicates hematoxylin and eosin; SPEP, serum protein electrophoresis; ANA, antinuclear antibody; ANCA, antineutrophilic antibody; ESR, erythrocyte sedimentation rate; CRP, C-reactive protein; HIV, human immunodeficiency virus. Asterisk indicates $\geq 80 \%$ of respondents reported routinely ordering; dagger, $60 \%-79 \%$ of respondents; double dagger, $40 \%-59 \%$ of respondents. 
Importantly, a stepwise diagnostic workup for PG is proposed based on survey results, which may limit unnecessary testing and the associated costs to the health care system (Figure 1). Selection of additional testing is guided by initial test results and features of the patient's clinical presentation, including age, review of systems, and associated comorbidities. Available data suggest that underlying inflammatory bowel disease is more frequent in PG patients who are younger than 65 years, whereas those who are 65 years and older are more likely to have inflammatory arthritis, cancer, or an underlying hematologic disorder. ${ }^{9}$

Treatment of PG should address both the inflammatory and wound components of the disease (Figure 2). ${ }^{7}$ In our survey results, systemic corticosteroids were identified as an important first-line therapy supported by reasonable evidence and were favored for their rapid response and minimal cost. ${ }^{1,10,11}$ Many respondents endorsed the use of systemic therapy in combination with topical steroids or calcineurin inhibitors. Combination therapy may provide more immediate control of rapidly progressing disease while minimizing adverse effects of long-term systemic corticosteroid use. A survey of German wound experts similarly endorsed frequent use of topical calcineurin inhibitors and combination systemic and topical glucocorticoid therapy as common therapeutic approaches. ${ }^{1}$

Importantly, treatments may vary depending on patient characteristics, comorbidities, and underlying disease, which underscores the need for individualized treatment approaches. Alternative first-line systemic treatments favored by respondents were cyclosporine, biologic medications, and antineutrophilic agents such as dapsone.

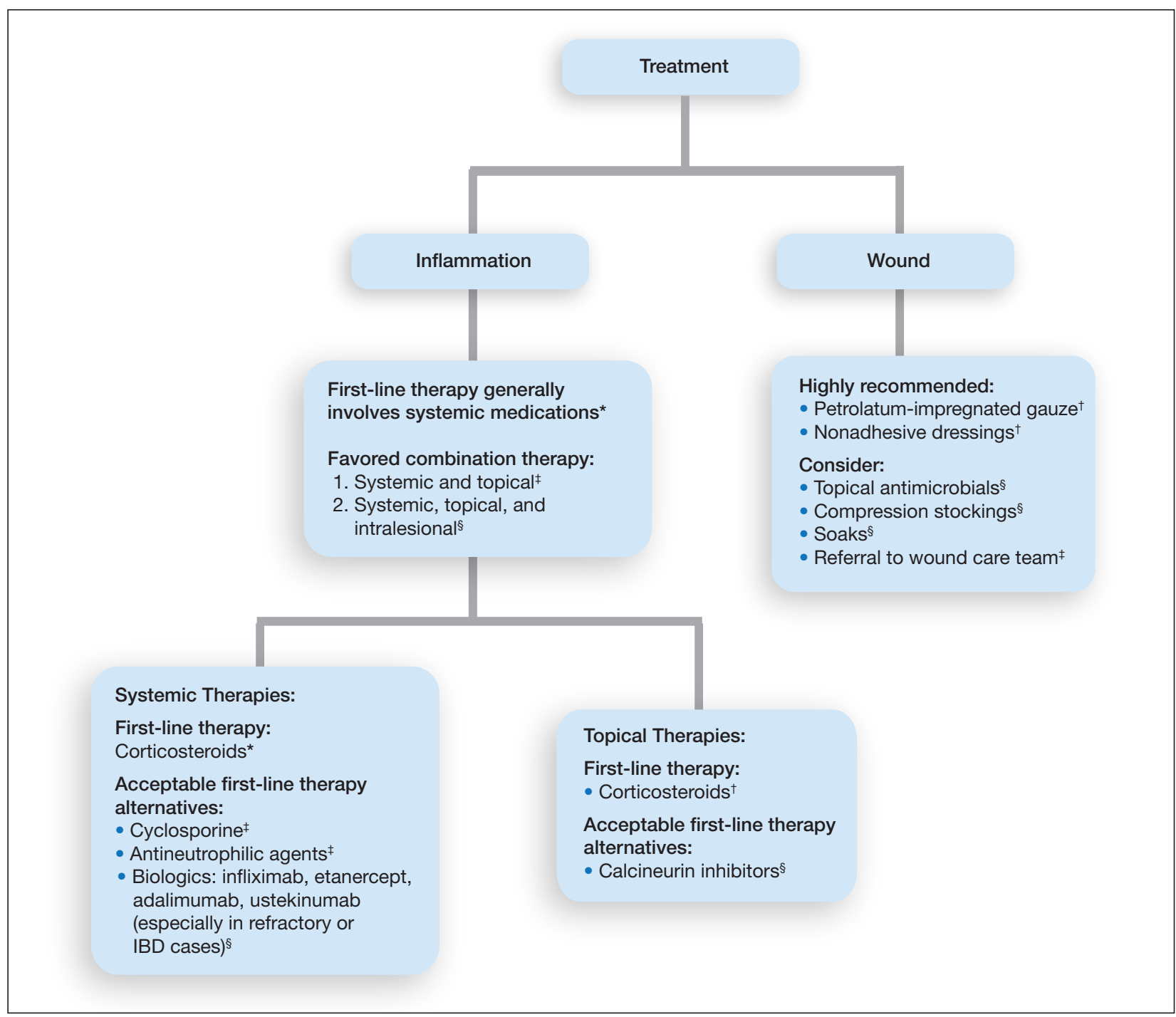

FIGURE 2. Proposed stepwise algorithm for the treatment of classic ulcerative pyoderma gangrenosum. IBD indicates inflammatory bowel disease. Asterisk indicates $\geq 90 \%$ of respondents reported routinely ordering; dagger, $60 \%-89 \%$ of respondents reported routinely ordering; double dagger, $40 \%-59 \%$ of respondents; section, $30 \%-39 \%$ of respondents. 
Cyclosporine has demonstrated comparable efficacy to systemic glucocorticoids in one RCT and is considered an important steroid-sparing alternative for PG treatment. ${ }^{2}$ Biologic agents, especially tumor necrosis factor inhibitors, may be effective in treating cases of refractory PG or for concomitant inflammatory bowel disease management, as demonstrated by a small RCT documenting improvement of PG following infliximab infusion. ${ }^{3}$

Respondents strongly recommended petrolatumimpregnated gauze and other nonadhesive dressings, including alginate and hydrocolloid dressings, as part of PG wound care. Topical antimicrobials and compression stockings also were recommended by respondents. These practices aim to promote moist environments for healing, avoid maceration, prevent superinfection, optimize wound healing, and minimize damage from adhesive injury. ${ }^{12}$ Wound debridement and grafting generally were not recommended. However, pathergy is not a universal phenomenon in PG, and wounds that are no longer in the inflammatory phase may benefit from gentle debridement of necrotic tissue and/or grafting in select cases. ${ }^{10}$

\section{Conclusion}

An approach to modifying PG management based on clinical presentation and the practice of combination therapy with multiple systemic agents in refractory PG cases was not addressed in our survey. The low response rate is a limitation; however, the opinions of 51 medical dermatologist experts who regularly manage PG (in contrast to papers based on individualized clinical experience) can provide important clinical guidance until more scientific evidence is established.

Acknowledgments - We would like to thank the SDH and RDS membership for their participation in this survey. We especially acknowledge the other members of the SDH Scientific Task Force for their feedback:
Misha Rosenbach, MD (Philadelphia, Pennsylvania); Robert G. Micheletti, MD (Philadelphia, Pennsylvania); Karolyn Wanat, MD (Milwaukee, Wisconsin); Amy Chen, MD (Cromwell, Connecticut); and A. Rambi Cardones, MD (Durham, North Carolina).

\section{REFERENCES}

1. Al Ghazal P, Dissemond J. Therapy of pyoderma gangrenosum in Germany: results of a survey among wound experts. J Dtsch Dermatol Ges. 2015; 13:317-324.

2. Ormerod AD, Thomas KS, Craig FE, et al. Comparison of the two most commonly used treatments for pyoderma gangrenosum: results of the STOP GAP randomised controlled trial. BMJ. 2015;350:h2958.

3. Brooklyn TN, Dunnill MG, Shetty A, et al. Infliximab for the treatment of pyoderma gangrenosum: a randomised, double blind, placebo controlled trial. Gut. 2006;55:505-509.

4. Al Ghazal P, Klode J, Dissemond J. Diagnostic criteria for pyoderma gangrenosum: results of a survey among dermatologic wound experts in Germany. J Dtsch Dermatol Ges. 2014;12;1129-1131.

5. Weenig RH, Davis MD, Dahl PR, et al. Skin ulcers misdiagnosed as pyoderma gangrenosum. N Engl J Med. 2002;347:1412-1418.

6. Powell FC, Su WP, Perry HO. Pyoderma gangrenosum: classification and management. J Am Acad Dermatol. 1996;34:395-409.

7. Bennett ML, Jackson JM, Jorizzo JL, et al. Pyoderma gangrenosum: a comparison of typical and atypical forms with an emphasis on time to remission. case review of 86 patients from 2 institutions. Medicine. 2000;79:37-46.

8. Su WP, Davis MD, Weening RH, et al. Pyoderma gangrenosum: clinicopathologic correlation and proposed diagnostic criteria. Int J Dermatol. 2004;43:790-800.

9. Aschyan H, Butler DC, Nelson CA, et al. The association of age with clinical presentation and comorbidities of pyoderma gangrenosum. JAMA Dermatol. 2018;154:409-413.

10. Binus AM, Qureshi AA, Li VW, et al. Pyoderma gangrenosum: a retrospective review of patient characteristics, comorbidities and therapy in 103 patients. Br J Dermatol. 2011;165:1244-1250.

11. Reichrath J, Bens G, Bonowitz A, et al. Treatment recommendations for pyoderma gangrenosum: an evidence-based review of the literature based on more than 350 patients. J Am Acad Dermatol. 2005;53:273-283.

12. Miller J, Yentzer BA, Clark A, et al. Pyoderma gangrenosum: a review and update on new therapies. J Am Acad Dermatol. 2010; 62:646-654. 


\section{APPENDIX}

\section{eTABLE 1. Survey Questions}

Demographic characteristics

What is your primary specialty?

Are you affiliated with a hospital?

Approximately how many patients with classic PG do you see in a year?

Diagnostic evaluation

Do you routinely perform a skin biopsy for H\&E in all your patients with possible diagnosis of classic PG?

Do you routinely perform a skin biopsy for tissue culture in all your patients with possible diagnosis of PG?

Do you order any of the following ancillary tests as part of your regular workup to establish the diagnosis of PG?

Do you routinely refer patients with classic PG to additional specialists?

Treatment

What is your first-line treatment approach for patients with classic PG?

Do you use a combination therapy approach as first-line treatment for patients with classic PG?

What topical therapy do you use as first-line therapy for patients with classic PG?

What systemic therapy do you use as first-line therapy for patients with classic PG?

Wound care

Do you routinely recommend the following for wound care management in patients with classic PG?

Do you routinely refer to a different specialty for wound care management of patients with classic PG?

Do you routinely recommend wound debridement for patients with classic PG?

Do you routinely recommend grafting over PG ulcers in patients with classic PG?

Abbreviations: PG, pyoderma gangrenosum; H\&E, hematoxylin and eosin. 
eTABLE 2. Diagnostic Evaluations for Pyoderma Gangrenosum ( $=51)$

\begin{tabular}{|c|c|}
\hline Evaluation & $\begin{array}{l}\text { Routinely Ordered, } \\
\mathrm{n}(\%)\end{array}$ \\
\hline Complete blood cell count & $49(96)$ \\
\hline Skin biopsy for H\&E & $47(92)$ \\
\hline Skin biopsy for tissue culture & $46(90)$ \\
\hline Complete metabolic panel & $44(86)$ \\
\hline Serum protein electrophoresis & $39(76)$ \\
\hline Hepatitis panel & $36(71)$ \\
\hline Rheumatoid factor & $35(69)$ \\
\hline Antinuclear antibodies & $34(67)$ \\
\hline Antineutrophilic antibodies & $33(65)$ \\
\hline Erythrocyte sedimentation rate & $30(59)$ \\
\hline C-reactive protein & $28(55)$ \\
\hline Cryoglobulins & $27(53)$ \\
\hline Urine protein electrophoresis & $27(53)$ \\
\hline Hypercoagulability workup ${ }^{b}$ & $25(49)$ \\
\hline Serum immunofixation test & $25(49)$ \\
\hline HIV serologic testing & $22(43)$ \\
\hline Chest radiograph & $21(41)$ \\
\hline Colonoscopy & $21(41)$ \\
\hline Gastroenterology referral & $19(38)$ \\
\hline Hematology/oncology referral & $7(14)$ \\
\hline
\end{tabular}

Abbreviations: H\&E, hematoxylin and eosin; HIV, human immunodeficiency virus.

aData represent a consolidation of respondents who answered, always performed or ordered (almost $100 \%$ of the time) and often performed or ordered (more than half the time).

bWorkup included fibrinogen, protein C/S, antithrombin III, lupus anticoagulant, anticardiolipin antibodies, beta-2 glycoprotein 1, factor $V$ Leiden, homocysteine, factor $V$ Leiden mutation, prothrombin 20210 mutation, and methylenetetrahydrofolate reductase mutation.

\section{eTABLE 3. Wound Care Management Recommendations for Pyoderma Gangrenosum ( $\mathrm{N}=51)$}

\begin{tabular}{ll}
\hline Recommendation & $\begin{array}{l}\text { Reported } \\
\text { Use, }{ }^{\mathrm{a}} \mathbf{n}(\%)\end{array}$ \\
\hline Petrolatum-impregnated gauze & $39(76)$ \\
\hline $\begin{array}{l}\text { Nonstick dressings (eg, hydrocolloid, } \\
\text { alginate) }\end{array}$ & $35(69)$ \\
\hline Topical antimicrobials & $22(43)$ \\
\hline Compression stockings & $20(39)$ \\
\hline $\begin{array}{l}\text { Soaks (eg, vinegar, aluminum sulfate } \\
\text { tetradecahydrate-calcium acetate, }\end{array}$ & $16(31)$ \\
\hline potassium permanganate) & \\
\hline Unna boot & $12(24)$ \\
\hline Enzymatic debridement agents (eg, & $8(16)$ \\
\hline collagenase) & $23(6)$ \\
\hline Biologic dressing (eg, composite, \\
dermal, epidermal)
\end{tabular}

aData represent a consolidation of respondents who answered always use (almost 100\% of the time) and often use (more than half the time). 